\title{
On the pulse of discovery
}

\author{
What started 50 years ago as a 'smudge' on paper has flourished into a fundamental field of astrophysics replete \\ with unexpected applications and exciting discoveries. To celebrate the discovery of pulsars, we look at the past, \\ present and future of pulsar astrophysics.
}

- B ifty years ago this month, Jocelyn Bell Burnell (pictured) had already seen the recurring blips on her hundredsof-metres-long chart paper that was being churned out by the newly minted 4-acrearray radio telescope at Cambridge, $\mathrm{UK}$. Together with her PhD advisor, Antony Hewish, they were hard at work trying to understand their nature and the source that could have generated them. The study of what we now know as pulsars has developed into a vibrant field of research that touches upon almost all areas of astrophysics and beyond: from stellar astronomy, to the physics of condensed matter, to gravitational waves and the very fabric of our Universe. In this focus issue we bring together some of the most exciting pulsar science and reflect on the future directions of the field.

The discovery of pulsars required the attentiveness of a self-professed impostersyndrome-suffering $\mathrm{PhD}$ student (whose contribution was disappointingly snubbed when the Nobel Prize committee came knocking), the prowess of a novel telescope probing new parts of parameter space and of course the collective thinking of our community to figure out what these blips were (see the Perspective by Jocelyn Bell Burnell). Pulsars are the end products of massive stellar evolution, compact stars made primarily out of neutrons, often spinning at dizzying speeds (up to $\sim 0.24$ times the speed of light) and showing a precision in their rotation, for the most stable pulsars, rivalling that of atomic clocks $\left(10^{-21} \mathrm{~s}\right)$. They also have prodigious magnetic fields, which, when combined with their rotation, lead to the emission of the pulses we observe.

Neutron stars were first predicted by Walter Baade and Fritz Zwicky in 1934 but the idea of a rotating neutron star with a strong magnetic field only arose in 1967, championed by Franco Pacini, and independently in 1968 by Thomas Gold, who even made the connection between such stars and the discovery reported by Hewish and Bell in the same year. The importance of this discovery for astrophysics and physics in general was perhaps made crystal clear by the discovery by Joseph
Taylor and Russell Hulse in 1975 of a pulsar in a binary system with another neutron star. The orbit of the pulsar was observed to shrink in the exact way that Einstein's general theory of relativity predicted if the system was emitting gravitational waves (and brought in another Nobel Prize for the pulsar field). Today, we are not only detecting pulsars and neutron stars from space (see the Mission Control by Keith Gendreau and Zaven Arzoumanian), but a network of pulsars is poised to become the largest gravitational-wave detector at our disposal (see the Article by Chiara Mingarelli and collaborators and the Comment by Andrea Lommen). Gravitational-wave detection aside, pulsars are now being used to probe the most fundamental properties of spacetime (see the Comment by Kuo Liu and Ralph Eatough) and of condensed matter. Some of these topics were discussed recently at the IAU Symposium 337 on the occasion of the pulsar discovery (summarized in the Meeting Report by Nanda Rea) that took place on the grounds of the Jodrell Bank Observatory - itself celebrating 60 years of discoveries in pulsar research and beyond (see the Perspective by Andrew Lyne and Ian Morison).

The discovery of pulsars was not only fortuitous but more importantly it was serendipitous. When the Cambridge radio astronomy group decided to build their telescope they had quasars in mind. This discovery is reminiscent of another discovery that heralded the era of precision cosmology - in 1964 Arno Penzias and Robert Wilson inadvertently detected the echo from the Big Bang. Fifty years later, the golden age of serendipitous discovery seems to be well and truly over. We find ourselves instead in the age of certainty, calculated risks, assured returns and zero-sum science (see the Correspondence by Maura McLaughlin). Have our telescopes become too expensive and our experiments too involved to allow room for the unexpected?

In some sense, whenever a truly new instrument is built (the Laser Interferometer Gravitational-wave Observatory springs to mind) or whenever we are

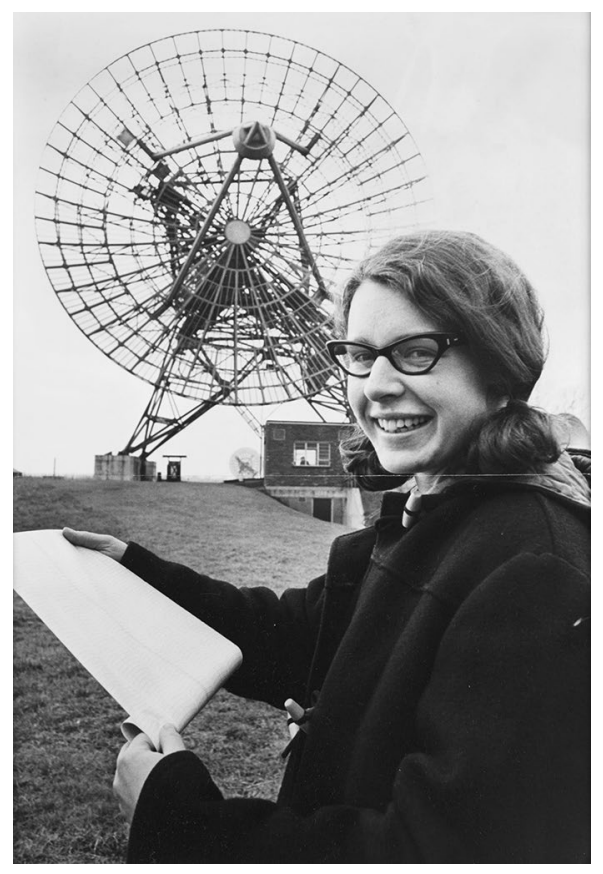

Credit: Daily Herald Archive / SSPL / Getty Images

probing a new niche of the phase space, serendipitous discoveries are bound to happen, as long as we have vigilance and an open mind to identify them as such - attributes Bell Burnell and Hewish did have. Nowadays, however, not many astronomers sift through piles of chart papers to identify signals. They instead build algorithms to do the sifting for them. How does one build intuitiveness into an algorithm, how does one teach algorithms to identify what is practically unknown? Unsupervised clustering from deeplearning neural networks may be a step in the right direction but this conundrum is something that deserves our community's undivided attention. Data from facilities like the Square Kilometre Array will soon be pouring into our servers and we need to be ready. Who knows what 'pulsars' may be hiding there?

Published online: 1 December 2017 https://doi.org/10.1038/s41550-017-0343-6 